\title{
Physical-Sensor and Virtual-Sensor Based Method for Estimation of Lower Limb Gait Posture Using Accelerometers and Gyroscopes*
}

\author{
Kun LIU**, Yoshio INOUE*** and Kyoko SHIBATA*** \\ ${ }^{* *}$ College of Mechanical Science and Engineering, Jilin University, Changchun China \\ E-mail:mikylk@hotmail.com \\ ***Department of Intelligent Mechanical Systems Engineering, Kochi University of Technology \\ 185 Miyanokuchi, Tosayamada-cho, Kochi Japan
}

\begin{abstract}
An approach using physical-sensor difference and virtual-sensor difference based algorithm to visually and quantitatively confirm lower limb posture was proposed and a wearable sensor system was developed. Flexion/extension (FE) and abduction/adduction (AA) hip joint angles and FE knee joint angle were estimated for orientations of the lower limb segments; the knee and ankle joint trajectories were calculated using the segmental orientation angles and lengths to estimate the positions of lower limb joints. In the wearable sensor system, an accelerometer on the hip and two $\mathrm{MAG}^{3} \mathrm{~s}$ (inertial sensor module) on the thigh were in grouped to measure the data for estimating the thigh orientation and knee joint position using the double-sensor difference based algorithm. Two $\mathrm{MAG}^{3} \mathrm{~s}$ on the thigh and shank near the knee joint were in grouped to measure the data for estimating the knee joint angle and ankle joint position using the virtual-sensor difference based algorithm. Compared with the camera motion capture system, the correlation coefficients in five trials were above 0.89 for the hip FE angle, higher than 0.9 for the hip AA angle and better than 0.88 for the knee FE angle. There was no integration of acceleration or angular velocity for the joint rotations and positions in this method. The developed wearable sensor system was able to visually and quantitatively confirm the lower limb posture with fewer sensors and higher accuracy than with other methods. It can be used as a substitute for the camera system for patient gait posture analysis in daily life.
\end{abstract}

Key words: Gait Posture, Wearable Sensor System, Visual Estimation, Virtual-Sensor

\section{Introduction}

Ambulatory assessment of gait posture is a promising clinical tool to diagnose walking disabilities. Especially 3D quantitative information for the lower limb gait posture is crucial for clinical evaluation and therapeutic treatment comparisons in the orthopedic and rehabilitation fields ${ }^{(1)}$. Tracking the position of body-mounted reflective markers using cameras is the most common method for gait posture analysis. In this method, motion information of hip and knee joints and lower limb segments can be derived, then the lower limb posture can be estimated. Estimation of the posture or motion of the human body using video or image data to extract parameters of human body model is being actively studied ${ }^{(2)(3)}$ Human joint motion for sports and medical purposes are also discussed ${ }^{(4)}$.

However, since a camera-based system is bulky, expensive and complex, it restricts the 
operators to a constrained environment where the cameras are installed so that it is not applicable for out-lab ambulatory measurement of under limb posture in the ordinary life. Recently, small inertial sensors combining accelerometers, gyroscopes and magnetometers were developed, which appeared to be promising for measuring human movement. Various alternative methods using inertial sensors were available for assessing 3D gait posture ${ }^{(5)(6)}$. Also, many ambulatory systems for various clinical applications have been developed to monitor physical activities ${ }^{(7-9)}$, for example, ambulatory systems for the quantitative and qualitative analysis of gait and posture in chronic pain patients treated ${ }^{(10)}$ or for the joint angle measurement ${ }^{(11)}$.

To estimate body segment orientation, integrating angular velocity of the body segments measured by gyro sensors has been a common method ${ }^{(12)}$, but the results are distorted by offsets and drifts in the data. Another method to estimate the hip and knee joint angles and positions for the 3D lower limb posture using the gravitational acceleration along the anterior axis of the segment was proposed ${ }^{(13)}$, however, the result was insensitive to the complex geomagnetic field distortions, and the application was limited to movement such as walking, running, or ascent and descent of stairs. An inertial sensor-based method for estimating knee joint FE angle using segmental acceleration and angular velocity data was described ${ }^{(14)}$. The method did not make use of the earth's magnetic field so it was also insensitive to the complex geomagnetic field distortions. However, it was only used for analyzing the knee joint FE angle - not the lower limb posture. A method for ambulatory measurement of 3D knee joint angles using both gyroscopes and accelerometers was presented (15) (16), which was expandable to other joints for the limb posture analysis. Another method for gait posture estimation using wearable accelerometer and gyro sensors was present ${ }^{(17)}$. Although the result showed that it was better for qualitative analysis than quantitative analysis, the experiments were conducted at fairly low velocity ( $88 \mathrm{steps} / \mathrm{min}$ ). Willemsen et al., ${ }^{(18)}$ gave a method that used only accelerometers for assessing the angle of lower extremities without integration, but the movements of the lower segment joints were only analyzed in the sagittal plane and thus two-dimensional. Dejnabadi et al., ${ }^{(19)}$ proposed a method to estimate sagittal kinematics of lower limbs orientation using body-fixed physical and virtual sensors, but there was no detailed application for the lower limb posture analysis. In addition, the fact that the physical sensors were attached on the frontal side of the thigh and shank resulted in a complicated algorithm to calculate the knee joint FE angle.

Therefore, a system which is accurate and easily used in routine practice to visually and quantitatively confirm the lower limb posture is greatly needed. The aim of our work is to propose a practical approach and develop an ambulatory system to analyze lower limb gait posture in daily life, without using gravitational acceleration and integration of angular velocity. The posture detection allows the assessment of hip joint and knee joint kinematics, the joint angles, positions and other temporal gait parameters (i.e. swing and stance).

We have recently presented a novel approach to ambulatory assessment of lower limb segmental orientation on a wearable sensor system ${ }^{(20)}$, in which only one type of sensor (accelerometer) was used and only arithmetic was adopted without integration. However, there was no further discussion of a gait posture analysis - only for the segmental orientation estimation. As a further research into our described work, the accelerations and angular velocities exerted on the lower limb segments were used to estimate the hip and knee joint angles, and the knee joint and ankle joint positions. The hip joint angles for the thigh orientation were estimated using double-sensor difference based algorithm. The knee joint rotational angle in the sagittal plane was calculated with virtual-sensor difference based algorithm. The lower limb posture was shown in the 3D coordinate frame. In order to ensure a reliable estimation of the lower limb posture, it was assumed that the lower limb segments were rigid segments, and the subjects walked in a straight forward way with very little trunk sway, skin artifacts and no significant IE rotation of the thigh and shank. 


\section{Materials and methods}

\subsection{Physical-sensor difference based algorithm for the estimation of hip joint angles}

Since the acceleration measured on a body segment contains gravitational acceleration, translational acceleration and rotational acceleration, the signal cannot be separated and is always a resultant signal composed of the three components. When two accelerometers are attached at two different positions with each corresponding axis in the same direction, the gravitational acceleration, translational acceleration, skin motion artifact and other noise acting on the two sensors should be the same except the rotational acceleration. To exploit the difference between the rotational accelerations, we have proposed a double-sensor difference based algorithm to estimate the rotational angles of lower limb segment in two directions for the segmental orientation in 3D space. In this method, as Fig. 1 shows, a rigid segment performs rotational trials in the local coordinate system Or-XrYrZr and translational motion in the global coordinate system $\mathrm{O}-\mathrm{XYZ}$, thereby simulating the motion of a thigh or shank when a person walks in a straight forward way without considering the trunk sway, skin artifacts and IE rotation of the leg. The FE angle $\theta_{\mathrm{FE}}$ and AA angle $\theta_{\mathrm{AA}}$ about the $\mathrm{Yr}$ and $\mathrm{Xr}$ axes in the local frame could be calculated as the Eq. (11) and (12) showed Ref. (20), which were used to describe the orientation of the thigh in the local coordinate frame and shown as follows.

$$
\begin{gathered}
\theta_{A A}=\sin ^{-1}\left(\frac{r_{B} a_{A y}-r_{A} a_{B y}}{\left(r_{B}-r_{A}\right) \sqrt{A_{O_{r} y}{ }^{2}+A_{O_{r} z}{ }^{2}}}\right)-\sin ^{-1}\left(\frac{A_{O_{r} y}}{\sqrt{A_{O_{r} y}{ }^{2}+A_{O_{r} z}{ }^{2}}}\right) \\
\theta_{F E}=\sin ^{-1}\left(\frac{r_{B} a_{A x}-r_{A} a_{B x}}{\left(r_{B}-r_{A}\right) \sqrt{A_{O_{r} x}{ }^{2}+A_{O_{r} y}{ }^{2}+A_{O_{r} z}{ }^{2}-\left(\frac{r_{B} a_{A y}-r_{A} a_{B y}}{r_{B}-r_{A}}\right)}}\right) \\
-\sin ^{-1}\left(\frac{A_{O_{r} x}}{\sqrt{A_{O_{r} x}{ }^{2}+A_{O_{r} y}{ }^{2}+A_{O_{r z}}{ }^{2}-\left(\frac{r_{B} a_{A y}-r_{A} a_{B y}}{r_{B}-r_{A}}\right)}}\right)
\end{gathered}
$$

where ( $A_{O_{r} x}, A_{O_{r} y}, A_{O_{r} z}$ ) is the measured acceleration of the local frame origin (hip joint) when the segment translates in the global frame. $\left(a_{A x}, a_{A y}, a_{A z}\right)$ and $\left(a_{B x}, a_{B y}, a_{B z}\right)$ are the accelerations measured at positions $\mathbf{A}$ and $\mathbf{B}, r_{A}$ and $r_{B}$ are the distances from the rotational joint to the positions $\mathbf{A}$ and $\mathbf{B}$ on the segment.

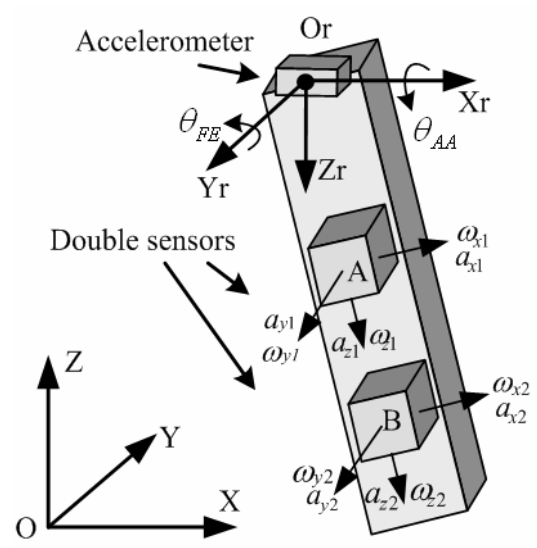

Fig. 1 Schematic diagram for the double-sensor difference based algorithm to calculate the orientation angles of a segment in the local coordinate system 


\subsection{Virtual-sensor difference based algorithm for the calculation of knee joint angle}

Dejnabadi et al. ${ }^{(19)}$ proposed a method to estimate sagittal kinematics of lower limb orientation using body-fixed physical and virtual sensors, but there was no further application for the lower limb posture analysis. Besides, in their method, the physical sensors were attached on the frontal side of the thigh and shank, which resulted to a complicated algorithm to calculate the knee joint FE angles. In this section, we proposed a simplified algorithm by attaching the physical sensors on the lateral surface of the thigh and shank segments. The main strategy for analyzing the knee joint angle between femur and tibia in the sagittal plane is to split the knee joint motion into linear translational motion of the knee joint and the rotational angular motion of the two segments about the knee joint. As Fig.2 shows, physical sensors are fixed at position $\boldsymbol{A}$ and $\mathbf{B}$ on the two linked segments and two virtual sensors are implanted at point $\boldsymbol{K}$. The rotational acceleration at position $\boldsymbol{A}$ on the segment $\boldsymbol{A K}$ is

$$
\ddot{\mathbf{r}}_{1}=\dot{\boldsymbol{\omega}}_{1} \times \mathbf{r}_{1}+\boldsymbol{\omega}_{1} \times\left(\boldsymbol{\omega}_{1} \times \mathbf{r}_{1}\right)
$$

and the acceleration $\mathbf{a}_{\mathbf{k} 1}$ at position $\boldsymbol{K}$ can be expressed using the following equation

$$
\mathbf{a}_{\mathrm{K} 1}=\mathbf{a}_{\mathrm{A}}+\ddot{\mathbf{r}}_{1}=\mathbf{a}_{\mathrm{A}}+\dot{\boldsymbol{\omega}}_{1} \times \mathbf{r}_{1}+\boldsymbol{\omega}_{1} \times\left(\boldsymbol{\omega}_{1} \times \mathbf{r}_{1}\right)
$$

Then the acceleration $\mathbf{a}_{\mathbf{k} 2}$ at position $\mathbf{K}$ can be expressed as follow if it calculated from the accelerations on the segment $\boldsymbol{K} \boldsymbol{B}$.

$$
\mathbf{a}_{\mathrm{K} 2}=\mathbf{a}_{\mathrm{B}}+\ddot{\mathbf{r}}_{2}=\mathbf{a}_{\mathrm{B}}+\dot{\boldsymbol{\omega}}_{2} \times \mathbf{r}_{2}+\boldsymbol{\omega}_{2} \times\left(\boldsymbol{\omega}_{2} \times \mathbf{r}_{2}\right)
$$

where $\mathbf{a}_{\mathbf{A}}$ and $\mathbf{a}_{\mathbf{B}}$ are the measured accelerations by the physical sensors at position $\boldsymbol{A}$ and $\boldsymbol{B}$, $\mathbf{a}_{\mathbf{k} 1}$ and $\mathbf{a}_{\mathbf{k} 2}$ represent the accelerations measured by the two virtual sensors at position $\boldsymbol{K}$.

The FE angle of the knee joint in the sagittal plane can be measured by the angle $\varphi$ between the femur and tibia (i.e., the rotational angle $\varphi$ between the two virtual sensors). Since one point should physically have a unique acceleration, the two virtual sensors on the knee joint must have equal accelerations in the same coordinate frame. Then the correction for coordinate frames rotation by angle $\varphi$ should be considered and the relationship between the accelerations of the two virtual sensors in the sagittal plane is:

$$
\mathbf{a}_{\mathrm{K} 1}=\mathbf{R}_{\varphi} \mathbf{a}_{\mathrm{K} 2}
$$

where $\mathbf{R}_{\varphi}$ is the rotation matrix. In the sagittal plane, the equation can be simplified,

$$
\left[\begin{array}{c}
a_{x_{A}}-r_{1} \omega_{1}^{2} \\
a_{y_{A}}+r_{1} \dot{\omega}_{1}
\end{array}\right]=R_{\varphi}\left[\begin{array}{c}
a_{x_{B}}-r_{2} \omega_{2}^{2} \\
a_{y_{B}}+r_{2} \dot{\omega}_{2}
\end{array}\right]
$$

and the rotational angle can be expressed as follow

$$
\varphi=\tan ^{-1}\left(\frac{a_{x_{K 2}}}{a_{y_{K 2}}}\right)-\tan ^{-1}\left(\frac{a_{x_{K 1}}}{a_{y_{K 1}}}\right)=\tan ^{-1}\left(\frac{a_{y_{B}}+r_{2} \dot{\omega}_{2}}{a_{x_{B}}-r_{2} \omega_{2}^{2}}\right)-\tan ^{-1}\left(\frac{a_{y_{A}}+r_{1} \dot{\omega}_{1}}{a_{x_{A}}-r_{1} \omega_{1}^{2}}\right)
$$

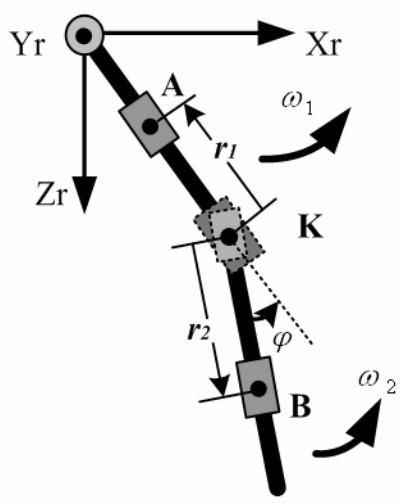

Fig.2 Virtual-sensor difference based method to calculate the knee joint angle in the sagittal plane. Two physical sensors were attached on the thigh and shank 


\subsection{Gait posture analysis of the lower limb}

In this part, to estimate the lower limb posture in the local coordinate system using the presented methods, a system is structured as Fig. 3 shows. To calculate the hip joint FE and AA angles and the knee joint trajectory using the double physical-sensor difference based algorithm, three physical sensors are in a group to measure accelerations and angular velocities. A physical sensor is fixed on the hip joint without rotation but translation only, and another two physical sensors with each corresponding axes in the same direction are attached on the thigh. Then to calculate the knee joint angle in sagittal plane and the ankle joint trajectory using the virtual-sensor difference based algorithm, two physical sensors are in a group to measure accelerations and angular velocities, which are attached near the knee joint on the thigh and shank. Table 1 shows the detailed terms used in the equations.

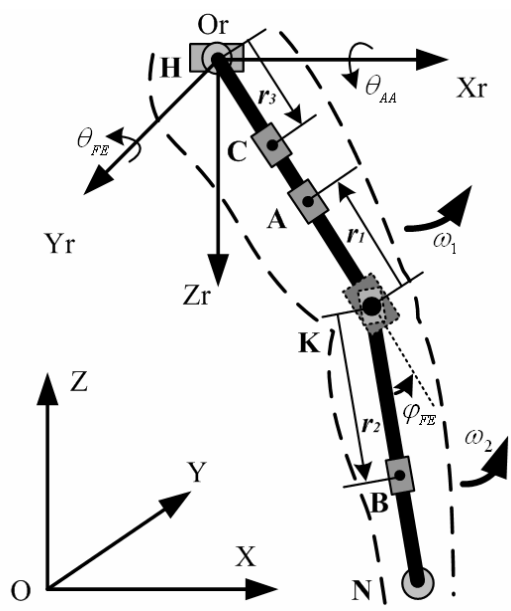

Fig.3 Lower limb model with sensors to estimate the joint rotations and positions

Table 1 List of the terms used in Fig.2

\begin{tabular}{|c|c|}
\hline Terms & Description \\
\hline$O-Z Y Z$ & Global coordinate system \\
\hline$O_{r}-Z_{r} Y_{r} Z_{r}$ & $\begin{array}{l}\text { Local coordinate system on the hip joint for the orientations of the lower limb segments (joint } \\
\text { rotations) and the trajectories of the lower limb joints (joint positions) }\end{array}$ \\
\hline$\theta_{A A}$ & Abduction/adduction angle of the thigh in the local coordinate system \\
\hline$\theta_{F E}$ & Flexion-extension angle of the thigh in the local coordinate system \\
\hline$\varphi_{F E}$ & Flexion-extension angle of the knee joint in the sagittal plane \\
\hline$r_{3}$ & Distance from hip joint to the physical sensor $\mathrm{C}$ on the thigh \\
\hline$r_{l}$ & Distance from the virtual sensor in the knee joint to the physical sensor A on the thigh \\
\hline$r_{2}$ & Distance from the virtual sensor in the knee joint to the physical sensor B on the shank \\
\hline
\end{tabular}

Then, to visually confirm the posture of the lower limb during walking, the positions of the hip joint, knee joint and ankle joint in the local coordinate system $\mathrm{Or}-\mathrm{XrYrZr}$ are calculated using the hip and knee joint angles and the segment lengths. The origin of the local coordinate system $\operatorname{Or}(0,0,0)$ was at the hip joint, then the coordinates for estimating the motions of knee and ankle joints were obtained with the following equations ${ }^{(13)}$.

$$
\begin{aligned}
& \mathbf{H}(x, y, z)=\left(\begin{array}{lll}
0 & 0 & 0
\end{array}\right) \\
& \mathbf{K}(x, y, z)=\left(\mathbf{R}_{\theta_{A A}} \mathbf{R}_{\theta_{F E}}\left(\begin{array}{c}
0 \\
0 \\
L_{\text {thigh }}
\end{array}\right)\right)^{\mathrm{T}} \\
& \mathbf{N}(x, y, z)=\left(\mathbf{R}_{\theta_{A A}} \mathbf{R}_{\theta_{\mathrm{FE}}}\left(\begin{array}{c}
0 \\
0 \\
L_{\text {thigh }}
\end{array}\right)\right)^{\mathrm{T}}+\left(\mathbf{R}_{\theta_{A A}} \mathbf{R}_{\theta_{F E}} \mathbf{R}_{\varphi_{F E}}\left(\begin{array}{c}
0 \\
0 \\
L_{\text {shank }}
\end{array}\right)\right)^{\mathrm{T}}
\end{aligned}
$$

where $L_{\text {thigh }}$ is the distance between the hip and knee joints, $L_{\text {shank }}$ is the distance between the knee and ankle joints, $\mathbf{R}_{\theta_{A A}}, \mathbf{R}_{\theta_{F E}}$ and $\mathbf{R}_{\varphi_{F F}}$ are rotation matrices 


\section{Experiment using the developed wearable sensor system}

In order to evaluate the presented approach, a wearable sensor system was developed. The system was mainly comprised out of one MCU (H8/3694, Renesas Technology Corp., Japan), one triaxial accelerometer-based chip (MM-2860, Sunhayato, Japan), and three analog inertial sensors named MAG $^{3}$ (MAG10-1200S050, MEMSense, USA) which could provide triaxial analog outputs of acceleration, rate of turn and magnetic field data. Fig. 4 shows the procedure of the experiment, and Fig.5 gives the configuration of the developed wearable sensor system.

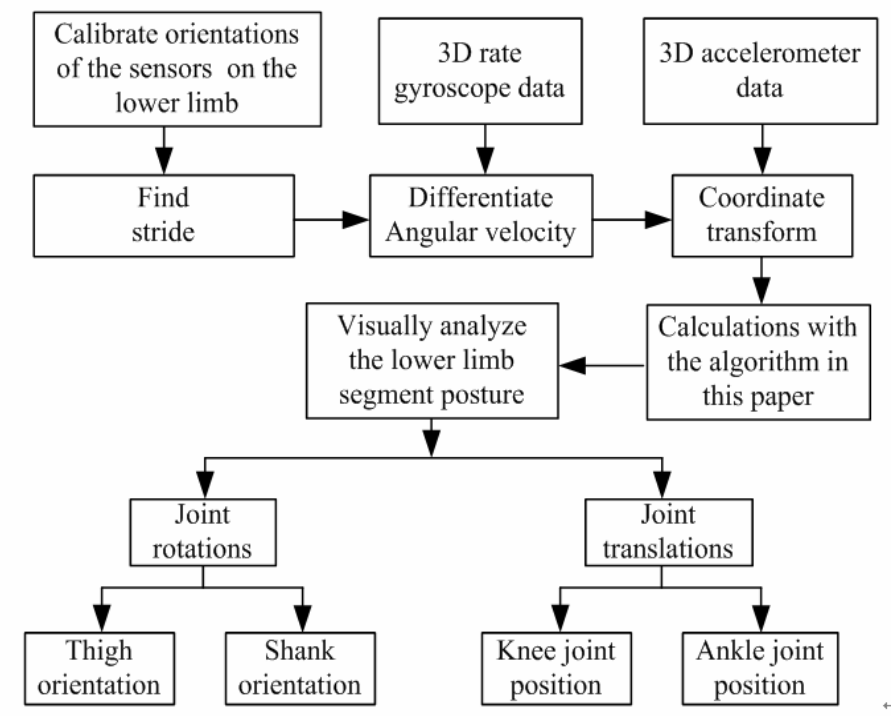

Fig.4 Schematic representation of the algorithm for deriving 3D thigh and shank orientations and the knee joint and ankle joint translations during gait on the basis of 3D inertial sensor signals.

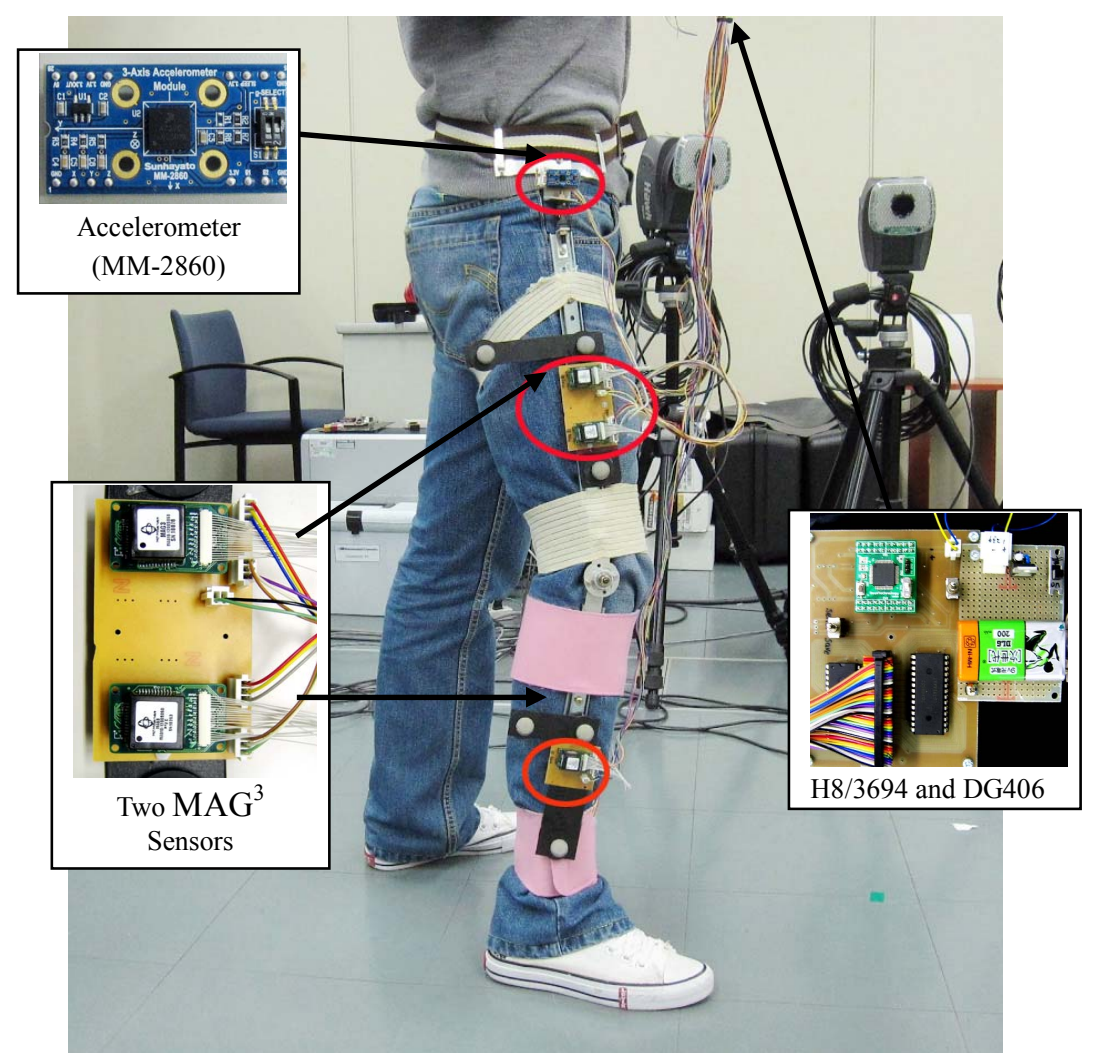

Fig.5 Experiment with the wearable sensor system in the working space of the optical motion system 
The two $\mathrm{MAG}^{3} \mathrm{~s}$ are integrated on a piece of electric circuit board and fixed on the thigh with corresponding axes in the same direction, at a distance of $60 \mathrm{~mm}$ from each other. A third $\mathrm{MAG}^{3}$ is attached on the shank. To prevent the skin motion artifact from adding noise to the measured accelerations and angular velocities, the sensors on the thigh and shank were fixed on one of the two stainless steel telescopic slide rails. The length of the slide rail can be adjusted according to the length of the thigh or shank of different subjects. To prevent gravity being erroneously recorded as acceleration in the horizontal plane and to ensure the measured accelerations coincided with the directions of idealized translational accelerations, the MM-2860 was fixed on a flat aluminum sheet, and was then attached on the hip joint. During the initial calibration, the aluminum sheet with the accelerometer was optimally adjusted to coincide with the sagittal plane of the thigh and the $\mathrm{z}$ axis of the accelerometer was upright. To ensure the thigh only performed FE and AA rotations without IE rotation as supposed in the method, the slide rail and the flat aluminum sheet were connected with an universal joint. Also the slide rails on the thigh and shank were connected using a bearing to make sure only FE rotation occurred in the knee joint. The slide rails were attached on the lateral surface of the lower limb with elastic straps, coinciding with the lines which connected the hip joint and knee joint, the knee joint and ankle joint. The recorded accelerations and angular velocities about $y$-axis indicated anterior-posterior motion, about $\mathrm{x}$-axis indicated lateral motion, and about $\mathrm{z}$-axis indicated vertical motion in the sensor coordination system on the thigh and shank.

In the experiment, five subjects ( 4 males, 1 females, Age: $25 \pm 3$ years, Height: $170 \pm 5 \mathrm{~cm}$, mass: $60 \pm 11 \mathrm{~kg}$ ) with no history of musculoskeletal pathology and injury were requested to perform 3 straight-line walking trials at self-selected walking speeds in each trial. Four groups of accelerations were simultaneously obtained from the wearable sensor system and a commercial optical motion analysis system, NAC Hi-Dcam II Digital High Speed Camera Systems (NAC image technology. Japan). The sampling frequency of the camera system is $100 \mathrm{~Hz}$, and the average calibration error is $0.014 \%$ for capturing stationary subjects and $0.21 \%$ for capturing dynamic subjects. Before the experiment, the objective and method of the experiment were explained to the subjects, and their written and oral consent to the experiment was obtained. This experiment has been approved by the ethics committee of the Department of Intelligent Mechanical Systems Engineering, Kochi University of Technology.

\section{Results}

All the readings of the sensors were recorded in the MCU real time and sent to PC off-line after each test, and the signals captured in the experiment were off-line processed. The sampling frequency was $100 \mathrm{~Hz}$ and the $\mathrm{A} / \mathrm{D}$ had a 12-bit resolution. The calculated rotation angles of the hip and knee joints were compared with the reference angles obtained from the camera system. Fig. 6 shows the compared result of the estimation for the FE and the AA angles of the hip joint, the FE angle of the knee joint in three trials by three subjects at self-selected speeds. The analysis result is shown in Table 2, where RMS was the root of the mean of the square differences, $\mathrm{R}$ was the correlation coefficient, $\mathrm{e}_{\max }$ was the maximum error. Since the trajectories of the knee and ankle joints were calculated from the three joint angles, the analysis result of the joint trajectories are not shown here - only the analysis result of the joint angles. Fig.7 shows trajectories of the knee and ankle joint in 3D coordination systems. Fig. 8 gives the continuous stick figures of the lower limb in one gait circle for visual confirmation of the human gait posture. 

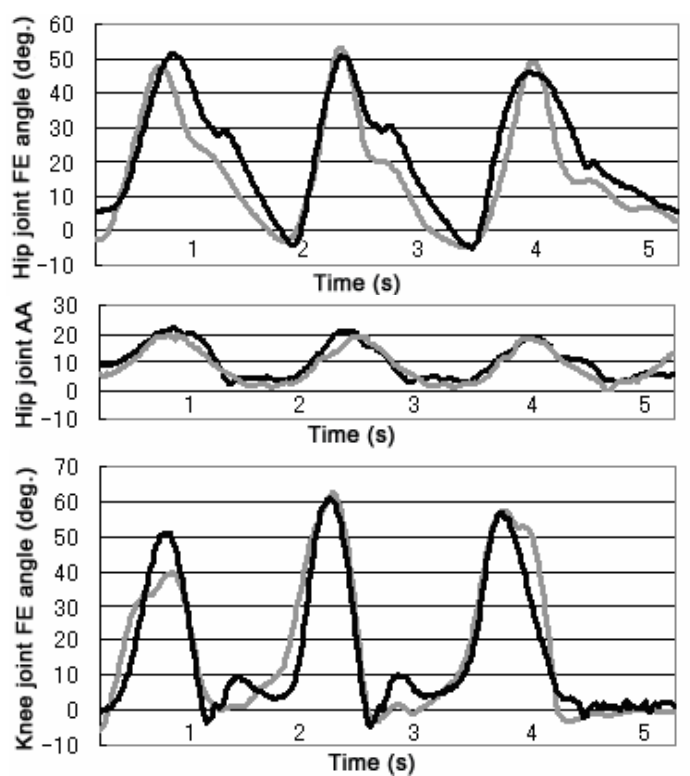

(a) Subject 1

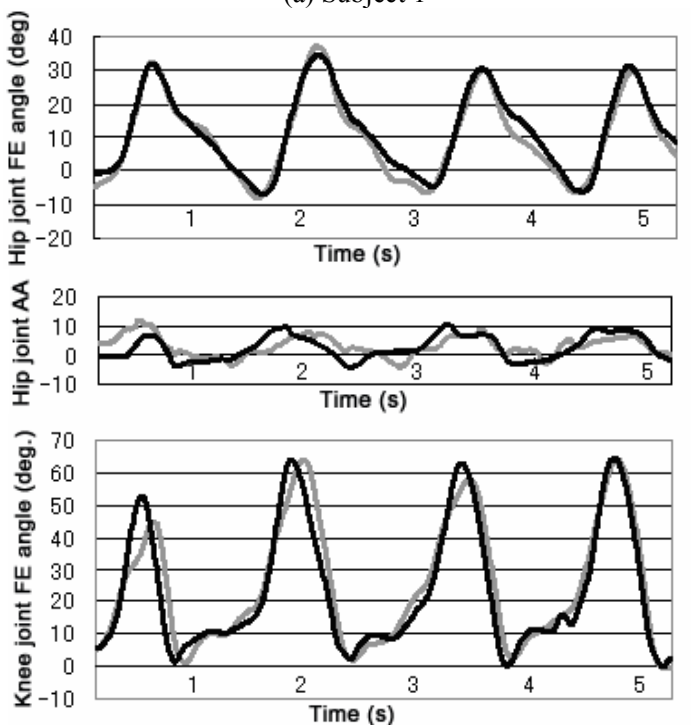

(b) Subject 2
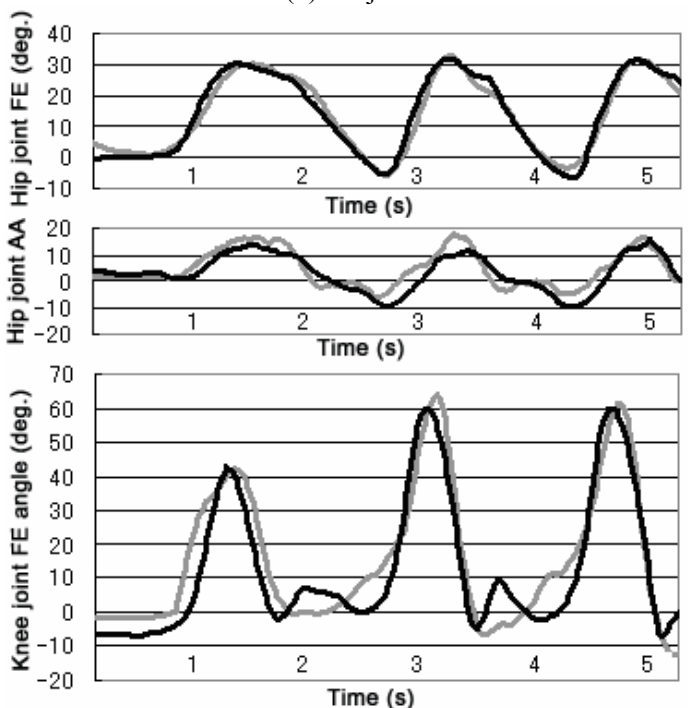

(c) Subject 3

Fig.6. The FE and AA angles of the hip joint and the FE angle of the knee joint in three trials by three different subjects at self-selected speeds. The dark black line was the referenced angle measured by the camera system and the light gray line was the calculated angle using measured signals measured by the developed wearable system 

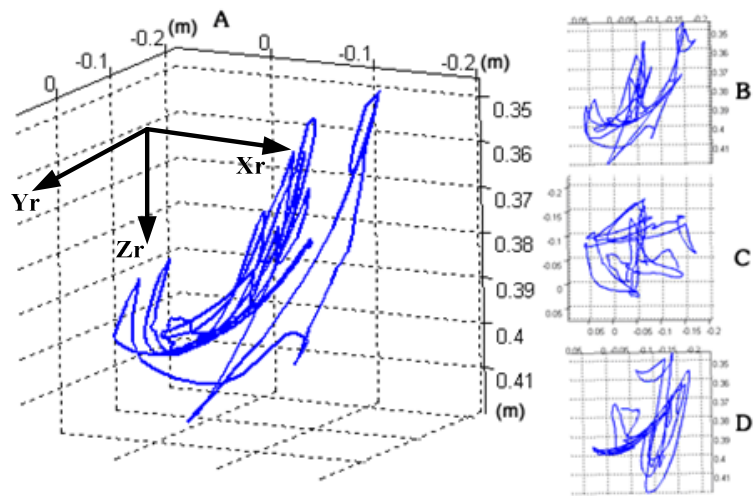

(a) Trajectory of the knee joint in 3D coordination systems
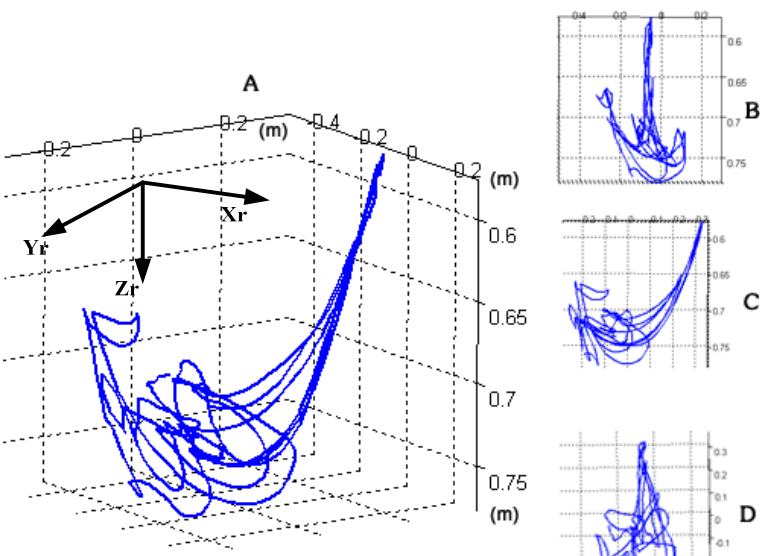

(b) Trajectory of the ankle joint in 3D coordination systems

Fig.7 To visually estimate the lower limb gait posture using the data obtained by the developed wearable system, the calculated trajectories of the knee and ankle joints in the 3D local coordinate system $(\mathrm{Or}$ - $\mathrm{Xr} Y r \mathrm{Zr})$ were showed on the left side marked as $\mathbf{A}$ in each picture. On the right side, the three smaller pictures marked as $\mathbf{B}, \mathbf{C}$ and $\mathbf{D}$ were the detail information of the joint trajectory in the sagittal, transverse and frontal plane.

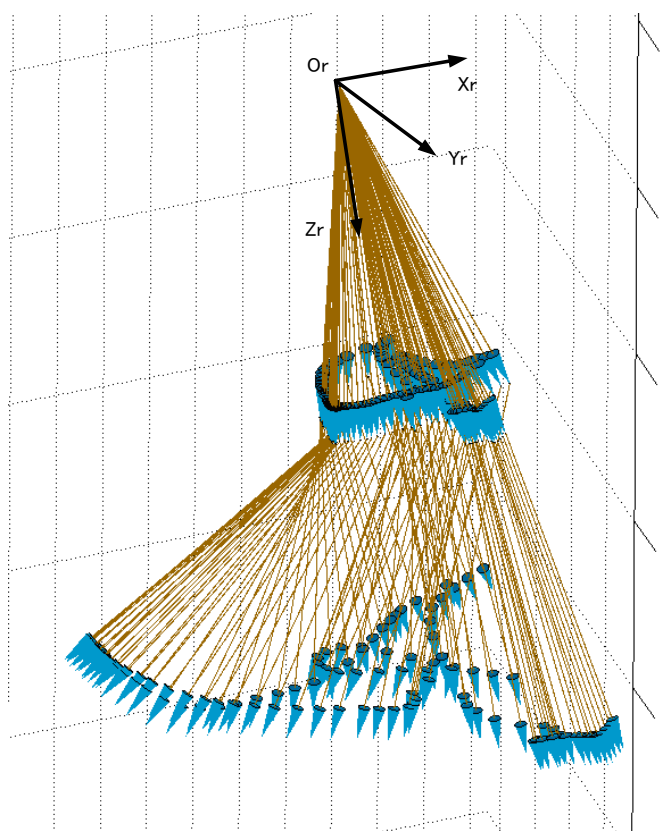

Fig.8 One gait circle shown by continuous stick figures to estimate the human gait posture. The blue arrows represent the knee joint and ankle joint. 
Table 2 Analysis result of the rotation angles of the hip and knee joints which were obtained from the referenced system and the wearable sensor system. The value of each parameter is an average of three trials when each subject walked at three self-selected speeds. The overall average of each parameter in five trials was given at the bottom.

\begin{tabular}{|c|c|c|c|c|c|c|c|c|c|}
\hline \multirow[t]{2}{*}{ Subject } & \multicolumn{3}{|c|}{$\begin{array}{c}\text { hip joint } \\
\text { FE angle } \theta_{F E}\end{array}$} & \multicolumn{3}{|c|}{$\begin{array}{c}\text { hip joint } \\
\text { AA angle } \theta_{A A}\end{array}$} & \multicolumn{3}{|c|}{$\begin{array}{l}\text { knee joint } \\
\text { FE angle } \varphi_{F E}\end{array}$} \\
\hline & RMS & $\mathrm{R}$ & $\mathrm{e}_{\max }$ & RMS & $\mathrm{R}$ & $\mathrm{e}_{\max }$ & RMS & $\mathrm{R}$ & $\mathrm{e}_{\max }$ \\
\hline 1 & 4.01 & 0.93 & 6.13 & 3.51 & 0.94 & 4.51 & 5.32 & 0.93 & 10.57 \\
\hline 2 & 3.28 & 0.94 & 6.53 & 3.78 & 0.95 & 3.94 & 4.31 & 0.95 & 6.21 \\
\hline 3 & 3.64 & 0.95 & 5.77 & 4.34 & 0.92 & 5.43 & 5.89 & 0.91 & 8.53 \\
\hline 4 & 5.33 & 0.89 & 10.12 & 4.93 & 0.9 & 6.75 & 6.69 & 0.88 & 10.42 \\
\hline 5 & 4.15 & 0.92 & 7.84 & 4.75 & 0.93 & 5.72 & 4.25 & 0.92 & 7.73 \\
\hline Average & 4.08 & 0.93 & 7.28 & 4.19 & 0.93 & 5.27 & 5.29 & 0.92 & 8.69 \\
\hline
\end{tabular}

\section{Discussion}

In this paper, we discussed an approach using physical-sensor difference and virtual-sensor difference based algorithm to estimate lower limb posture. Since the flexion-extension rotation is the main rotation of the three rotations (FE, AA and IE) of the knee joint, to simplify the algorithm, we only considered the FE rotation of the knee joint in this paper. However, the AA and IE rotation, which were ignored, must contaminate the measured signals and affect the calculated result of the FE rotation angle. To ensure there was no AA and IE rotation of the knee joint, we fixed the sensors on stainless rails connected with a bearing. Therefore it was one DOF analysis of the knee joint and that is why the error of the knee joint FE angle is large. Another limitation of the method was the assumption of no rotation of the trunk but only straight forward translation in the walking direction. Therefore, the method introduced here was based on the assumption that the accelerations measured on the hip joint only included translational and gravitational accelerations without rotational accelerations.

The analysis results in Table 2 showed that the presented method was strongly correlated with the camera system data and involved significantly less calculation than reported in the Ref. (17). The method was suitable for various walking velocities in the sagittal plane, whether the anterior/posterior acceleration of the trunk increased or not. However, Takeda et al. ${ }^{(13)}$ neglected any anterior/posterior acceleration and only conducted experiments at fairly low velocity ( $88 \mathrm{steps} / \mathrm{min})$. Therefore, it was an improvement on the method described in Ref. (13). Compared with the result in Table 3 of Ref. (13) and in Table 2 of this paper, the RMS in this paper is smaller and the R is greater than those in Ref. (13). Therefore, the result in this paper was better than that which Takeda et al. presented.

In addition, since there must be skin motion artifact due to impact loading and muscle activation, the attachment of any kind of wearable sensor on the lower limb segments could induce error. And in the experiment, the wearable sensor system was tested on volunteers with trousers, which must induce error also. Especially during the swing phase and at the time the foot contacted the floor when the volunteer was walking at a high speed, the sensors on the slide rails must have had relative motion with the thigh and shank, which would have contaminated the measured accelerations and angular velocities, thus reducing error to the calculated result.

Besides, the increase of non-translational acceleration of the trunk along with the increase of the walking speed also contaminated the accelerations measured by the accelerometer on the hip. Furthermore, in the initial calibration, since the accelerometer on the hip could not be placed exactly in the upright, forward and outward directions as assumed, the gravitational acceleration also contaminated the measured accelerations about each axis. Therefore the measured acceleration of the hip joint, assumed as translational acceleration only and used for the calculation in the algorithm, must have induced error to the result.

To control these problems, rigorous initial calibration process and predefined motions were conducted in each trial. After the sensor system was worn on the lower limb, all the 
sensors were aligned upright in the sagittal plane. However with the limitations detailed here, the work shows that the wearable sensor system can provide quantitative analysis of the lower limb gait posture with high accuracy, which was expressed by joint angles for lower limb segment orientations and joint trajectories for lower limb joint positions. To provide more detailed information for the gait posture analysis, further research is under way to develop a method for calculating the IE rotation of the hip joints and more rotations of the knee joint.

\section{Conclusion}

The combination of physical-sensor difference based algorithm and virtual-sensor difference based algorithm was original and created for the analysis of lower limb posture. There was no integration of angular acceleration or angular velocity in the method. The developed wearable sensor system was suitable for estimating the absolute hip joint FE and AA angles and knee joint FE angle in the local coordinate system, and the joint trajectories for the 3D lower limb posture analysis. It was reliable and convenient to do ambulatory, visual and quantitative estimation of lower limb gait, therefore it can be used as a substitute for the camera system to analyze gait posture of patients or healthy persons in daily life. The method can be easily utilized to estimate the posture of other segments such as upper limb or mechanical arms.

\section{Acknowledgement}

The authors wish to acknowledge the support of the volunteer subjects of Robotics and Dynamics Research Lab in Kochi University of Technology

\section{References}

(1) de Asla RJ, Kozanek M., Wan L., Rubash HE., Li G., Six DOF in vivo kinematics of the ankle joint complex: application of a combined dualorthogonal fluoroscopic and magnetic resonance imaging technique, Journal of Orthopaedic Research, Vol.24, No.5(2006), pp.1019-1027.

(2) Iwasawa, S., Ebihara, K., Ohya, J., Morishima, S., Real-Time Estimation of Human Body Posture from Monocular Thermal Images. IEEE Computer Society Conference on Computer Vision and Pattern Recognition, (1997), pp. 15-20.

(3) Takahashi, K., Sakaguchi, T., Ohya, J., Real-time estimation of human body postures using Kalman filter. 8th IEEE International Workshop on Robot and Human Interaction, (1999), pp.189-194.

(4) Ahn Ryul Choi, Yong Hoon Rim, A position based kinematic method for the analysis of human gait, Journal of Mechanical Science and Technology, Vol.19, No.10 (2005), pp.1919-1931.

(5) Christine Azevedo, et al., Rehabilitation of Functional Posture and Walking-Coordination of Healthy and Impaired Limbs, Journal of Automatic Control, No.15(2005), pp. 11-14.

(6) Gallagher, A., Matsuoka, Y., Wei-Tech Ang, An efficient real-time human posture tracking algorithm using low-cost inertial and magnetic sensors, International Conference on Intelligent Robots and Systems (IROS 04), (2004), pp. 2967-2972.

(7) Pages, G., Ramdani, N., Fraisse, P., Guiraud, D., Upper body posture estimation for standing function restoration, IEEE International Conference on Robotics and Automation (ICRA 07), 2007, pp. 3742-3747.

(8) Plamondon A., Delisle A., Larue C., et al., Evaluation of a hybrid system for three-dimensional measurement of trunk posture in motion, Applied Ergonomics, Vol.38, No.6(2007), pp. 697-712. 
(9) Salarian, A., Russmann, H., Wider, C. et al., Quantification of tremor and bradykinesia in Parkinson's disease using a novel ambulatory monitoring system. IEEE Transactions on Biomedical Engineering, Vol.54, No. 2(2007), pp. 313-322.

(10) Paraschiv-Ionescu A., Buchser E. E., Rutschmann B., Najafi B., Aminian K., Ambulatory system for the quantitative and qualitative analysis of gait and posture in chronic pain patients treated with spinal cord stimulation, Gait \& Posture, Vol.20, No. 2(2004), pp. 113-125.

(11)Karol J. O’Donovan, Roman Kamnik, Derek T. O’Keeffe, Gerard M. Lyons, An inertial and magnetic sensor based technique for joint angle measurement, Journal of Biomechanics, Vol.40, No.12(2007), pp. 2604-2611.

(12) Dejnabadi, H., Jolles, Brigitte M., Casanova, Emilio, Fua, Pascal, Aminian, Kamiar, Estimation and visualization of sagittal kinematics of lower limbs orientation using body-fixed sensors, IEEE Transactions on Biomedical Engineering, Vol.53, No.7(2006), pp. 1385-1392.

(13) Takeda Ryo, Tadano Shigeru, Todoh Masahiro, Morikawa Manabu, Nakayasu Minoru, Yoshinari Satoshi, Gait posture estimation using wearable acceleration and gyro sensors, Journal of Biomechanics, Vol.42, No.15 (2009), pp.2486-2494.

(14) Glen Cooper, Ian Sheret, Louise McMillian, Konstantinos Siliverdis, Ning Sha, Diana Hodgins, Laurence Kenney, David Howard, Inertial sensor-based knee flexion-extension angle estimation, Journal of Biomechanics, Vol.42, No.16 (2009), pp. 2678-2685.

(15) Favre, J., Aissaoui, R., Jolles, B.M., de Guise, J.A., Aminian, K., Functional calibration procedure for 3D knee joint angle description using inertial sensors, Journal of Biomechanics, Vol.42, No.14 (2009), pp. 2330-2335

(16) Favre, J., Jolles, B.M., Aissaoui, R., Aminian, K., Ambulatory measurement of 3D knee joint angle, Journal of Biomechanics, Vol.41, No.5 (2008), pp. 1029-1035.

(17) Takeda Ryo, Tadano Shigeru, Todoh Masahiro, Morikawa Manabu, Nakayasu Minoru, Yoshinari Satoshi, Gait analysis using gravitational acceleration measured by wearable sensors, Journal of Biomechanics, Vol.42, No.3 (2009), pp. 223-233.

(18) Willemsen T. M., Frigo C. and Boom H. B. K., Lower extremity angle measurement with accelerometers-error and sensitivity analysis, IEEE Transactions on Biomedical Engineering, Vol.38, No.12 (1991), pp. 1186-1193.

(19)Dejnabadi H., Jolles B. M., and Aminian K., A new approach to accurate measurement of uniaxial joint angles based on a combination of accelerometers and gyroscopes, IEEE Transactions on Biomedical Engineering, Vol.52, No.8 (2005), pp. 1478-1484.

(20) Liu Kun, Liu Tao, Shibata Kyoko, Inoue Yoshio, Zheng Rencheng, Novel approach to ambulatory assessment of human segmental orientation on a wearable sensor system, Journal of Biomechanics, Vol.42, No.16 (2009), pp. 2747-2752. 